\title{
Editorial Notice
}

I am pleased to announce the appointment of two new Associate Editors for the Journal. They are Dr E. Infeld, of the Institute of Nuclear Research, Warsaw, Poland, and Professor P. K. Kaw, of the Institute for Plasma Research, Bhat, India. They will both be very welcome additions to the Editorial Team. They and their work will be already well known to many of our readers and contributors.

At the same time, Professor F. D. Kahn has indicated his wish to leave the team, so on behalf of myself and the plasma physics community, I take the opportunity to thank him warmly for serving for some twenty years.

\section{J. P. Dougherty}

